\title{
Human-Robot Physical Interaction and Collaboration using an Industrial Robot with a Closed Control Architecture
}

\author{
Milad Geravand Fabrizio Flacco Alessandro De Luca
}

\begin{abstract}
In physical Human-Robot Interaction, the basic problem of fast detection and safe robot reaction to unexpected collisions has been addressed successfully on advanced research robots that are torque controlled, possibly equipped with joint torque sensors, and for which an accurate dynamic model is available. In this paper, an end-user approach to collision detection and reaction is presented for an industrial manipulator having a closed control architecture and no additional sensors. The proposed detection and reaction schemes have minimal requirements: only the outer joint velocity reference to the robot manufacturer's controller is used, together with the available measurements of motor currents and joint positions. No a priori information on the robot dynamic model and existing low-level joint controllers is strictly needed. A suitable on-line processing of the motor currents allows to distinguish between accidental collisions and intended human-robot contacts, so as to switch the robot to a collaboration mode when needed. Two examples of reaction schemes for collaboration are presented, with the user pushing/pulling the robot at any point of its structure (e.g., for manual guidance) or with a compliant-like robot behavior in response to forces applied by the human. The actual performance of the methods is illustrated through experiments on a KUKA KR5 manipulator.
\end{abstract}

\section{INTRODUCTION}

Safe physical Human-Robot Interaction (pHRI) typically requires lightweight and compliant mechanical structures, external sensing capabilities, and effective control schemes so as to prevent collisions and/or address the various phases of an impact, i.e., collision detection and robot reaction [1], [2]. These robot characteristics should be able to handle both unexpected collisions and intentional contacts, minimizing the risk of injuries in the first case [3] and establishing useful human-robot collaboration in the latter [4].

To address the mechanical issues of safe pHRI, some research robots, such as the series of DLR LWR manipulators [5] or the Barrett WAM [6], have been designed by introducing on purpose compliant joints [7], [8] (recently, even with variable stiffness [9]) and adopting slender and light mechanical links. In particular, the technology of the DLR LWR-III arm has been recently transferred to an industrial product, the KUKA LWR4+ robot.

For collision avoidance, different types of exteroceptive sensors are used to monitor the robot workspace and a large variety of control schemes have been proposed to

The work was performed while all authors were at the Dipartimento di Ingegneria informatica, automatica e gestionale Antonio Ruberti, Università di Roma "La Sapienza", Via Ariosto 25, 00185 Rome, Italy. The first author is now with the Institute of Automatic Control Engineering, TUM, Germany (milad.geravand@tum.de, \{fflacco,deluca $\} @$ dis.uniroma1.it). This work is supported by the European Commission, within the FP7 ICT-287513 SAPHARI project (www.saphari.eu) guarantee co-existence of a robot and a human operator see, e.g., [10]-[12].

Much attention has been devoted to the basic problem of detecting a physical collision between the manipulator and its environment, using only proprioceptive sensors. The classical approach is based on recognizing abnormal variations of the motor currents driving the robot, treated as actuation faults [13]. This feature is present also in some industrial robots, such as $\mathrm{ABB}$ manipulators running the proprietary control software IRC5 [14]. However, collision detection without its further isolation (i.e., recognizing which link has collided) allows only an immediate stop of the robot after the impact - the simplest robot reaction strategy. Advanced model-based methods, using an adaptive impedance control scheme [15] or monitoring the robot generalized momentum [16]-[18], are able instead to extract more information from a physical collision. In particular, the method in [16], [17] efficiently estimates the actual joint torques due to collision at a generic location along the manipulator through a residual vector signal, without the need of joint torque sensing. In turn, this allows the design of active/directional reaction strategies that safely push the robot away from the collision area.

A further step in pHRI research is concerned with collaboration. In this context, a main challenge is to distinguish between accidental collisions and intentional contacts, the latter being associated to the human intention to start a physical collaboration phase. A control architecture that integrates collision avoidance, detection, and reaction capabilities, as well as human-robot collaboration, has been recently presented in [19]. Additional work in this direction is the subject of the on-going European project SAPHARI.

Beside using innovative mechanical/actuation designs and possibly involving extra sensors, the above collision detection and reaction methods rely on two specific operative conditions: $i)$ the availability of a reliable robot dynamic model, which is used for residual computations; ii) the accessibility to motor torque/current commands, which can be modified on line under strict real-time constraints. However, wishing to realize sensor-less collision detection and reaction also on conventional industrial manipulators, both the above conditions fail to be satisfied. In fact, most industrial robots come with a closed control architecture that allows only kinematic control: the end-user can only modify the outer velocity or position references to the low-level joint controllers. Moreover, no information on the dynamic robot model is typically available, and even the structure and parameters of the joint-level inner control loops are unknown. 
The main goal of this paper is to present and evaluate an approach to collision handling in pHRI for industrial robots with a closed control architecture. No a priori knowledge is assumed about the robot dynamic model and the lowlevel controllers. As a paradigmatic example, we consider a small-size 6R KUKA manipulator, in which joint velocity references can be changed by a user-defined program through the communication interface available from the robot manufacturer. The interface outputs every $12 \mathrm{~ms}$ the actual joint encoder measures and a signal related to the motor currents.

By processing the current measurements during robot motion and comparing them with time-varying thresholds that depend on the commanded joint trajectory, whole-body collision detection can be realized. To improve sensitivity, we first eliminate from the measured currents the configurationdependent part accounting for gravity, which is identified in advance through static experiments. Furthermore, by separately high-pass and low-pass filtering of the motor currents, it is possible to distinguish between accidental collisions and intentional soft contacts with a human. When an intentional contact is recognized, the robot stops and switches to a collaboration mode. Two examples of robot reactive behavior in human-robot collaboration are presented, one with the human manually driving the robot by pushing/pulling it at any point of the structure, another with the robot realizing a compliant-like reaction to an instantaneous force applied (anywhere) by the human. To obtain these behaviors, we use directional information in the joint space obtained by measuring the small joint position variations occurring when the user applies a force in static conditions.

The paper is organized as follows. We present the operating conditions on our KUKA KR5 robot in Sect. II, including a gravity identification scheme that works on the motor currents. In Sect. III, the proposed collision detection method and the use of current filtering for distinguishing collisions and intentional contacts are described and validated through experiments. The robot reactive behaviors are presented and tested in Sect. IV. The paper is accompanied by a video attachment illustrating the performance of the approach.

\section{The KuKa KR5 Robot System}

The proposed collision detection and reaction schemes have been implemented on a KUKA KR 5 sixx R650 industrial robot available in our Robotics Lab, see Fig. 1. This is a small-size $6 \mathrm{R}$ manipulator with a spherical wrist, having $28 \mathrm{~kg}$ of weight for the moving parts, $5 \mathrm{~kg}$ of payload, and maximum stretch of $0.855 \mathrm{~m}$ from the base. The robot uses a KUKA KR C2sr controller that implements low-level motor control laws and motion control in the joint or Cartesian space. The control architecture is actually closed to the enduser, who can program the robot through the KCP teach pendant or using the proprietary KRL language and a humanmachine interface. When the robot is equipped with the KUKA Robot Sensor Interface (RSI) [21], control software can be implemented on an external $\mathrm{PC}^{1}$ that communicates with the KUKA controller every $12 \mathrm{~ms}$, possibly collecting also data from exteroceptive sensors (e.g., vision, depth sensor, force/torque sensor).
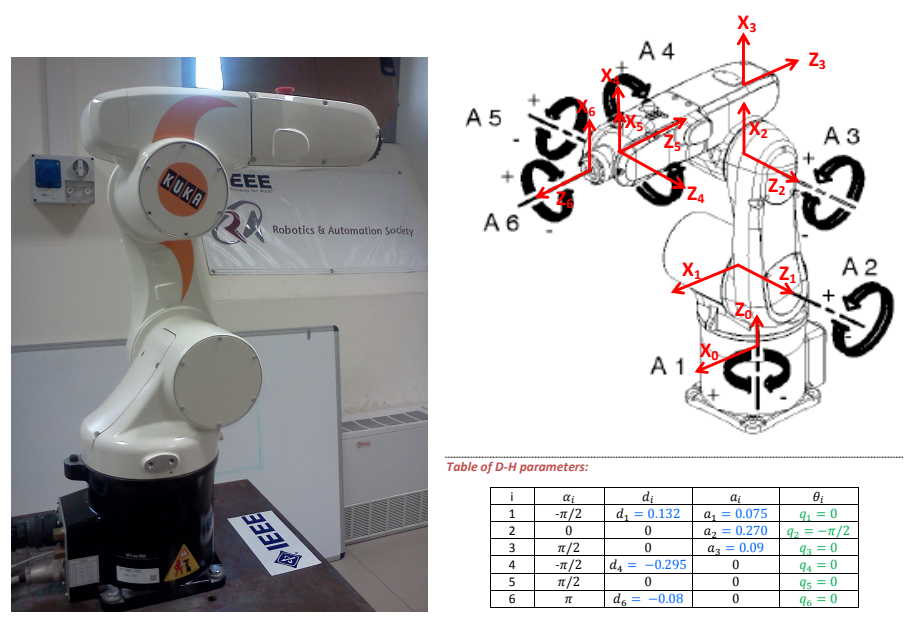

Fig. 1. The KUKA KR5 robot and the used D-H frames and table

With reference to Fig. 2, the input provided to the KUKA controller is typically in the form of velocity or position references at the joint level, respectively $\dot{\boldsymbol{q}}_{r}$ or $\boldsymbol{q}_{d}$. The available output from the robot system consists of the joint position $\boldsymbol{q}$, measured by encoders, and the (absolute value of) applied motor current $i$.

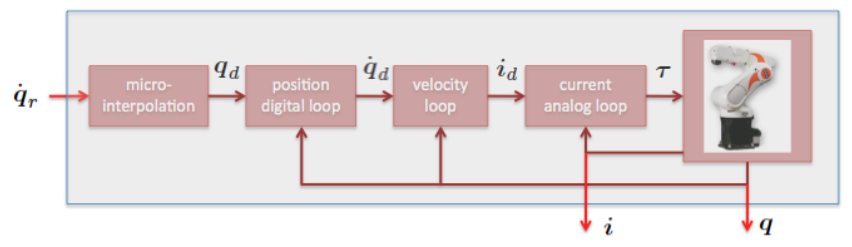

Fig. 2. A generic block diagram for the joint position, velocity, and current loops embedded at the low level of an industrial robot controller

We note the following:

1) The control block diagram in Fig. 2 is a generic one. Though reasonable, it may not correspond necessarily to the one used in KUKA robots. As a matter of fact, we do not have any information neither on the lowlevel control structure nor on the value of the control parameters. In particular, the command torque $\tau$ is not measured by any sensor and also the motor current-totorque gain is unknown.

2) The performance of any user-defined robot monitoring or control scheme is hampered by the relatively slow sampling time ( $T=12 \mathrm{~ms}$ ) of the communication allowed by the RSI interface. Nonetheless, since the sampling rate of the low-level KUKA digital controller is much higher (and due to the possible presence of

\footnotetext{
${ }^{1}$ We used an Intel Core 2 Quad Q6600@2.40GHz, with 2Gb of RAM, under real-time operative system Ubuntu patched with Real Time Application Interface (RTAI) for Linux.
} 
analog current loops), a rather accurate reproduction of velocity reference commands $\dot{\boldsymbol{q}}_{r}$ is obtained, at least at moderate speed.

3) According to the robot manufacturer, the RSI interface provides to the user only the absolute value $\left|i_{j}\right|$ of the motor currents, for $j=1, \ldots, 6$. In principle, this is enough for checking the occurrence of faulty situations for the motors and the system. Unfortunately, this limited information complicates the use of currents for detecting collisions (and their directional effect at the level of robot joints), as well as for the task of identifying the robot dynamic model.

To illustrate the above items 2) and 3), we present an illustrative experiment performed on the first three joints of the KUKA KR 5 robot $^{2}$. The reference motion for each joint is specified as

$$
\dot{q}_{r, j}(t)=A_{j} \cos \omega\left(t-t_{0}\right), \quad \text { for } j=1,2,3, \quad t \geq t_{0},
$$

with the motion starting at $t_{0}$ and where $\omega=1.306[\mathrm{rad} / \mathrm{s}]$, $A_{1}=-40, A_{2}=15$, and $A_{3}=20[\mathrm{deg} / \mathrm{s}]$. Figure 3 shows $^{3}$ the commanded reference velocity $\dot{\boldsymbol{q}}_{r}$, the resulting measured position $\boldsymbol{q}$, and the joint velocity $\dot{\boldsymbol{q}}$ reconstructed by offline numerical differentiation (using 5 position data centered around the current sample). Despite a delay of about $84 \mathrm{~ms}$ (7 samples) is present between $\dot{\boldsymbol{q}}_{r}$ and $\dot{\boldsymbol{q}}$, this affects mainly the performance of user-defined laws acting through the external communication/control loop. On the other hand, the overall performance of the KUKA internal controller appears to be satisfactory, with the low-level control loops overcoming most of the coupled and nonlinear dynamics of the robot.

Figure 4 shows the motor currents associated to the previous robot motion, as provided by the RSI. Despite an oscillatory motion is being commanded to the joints, these signals are indeed always positive. They contain relevant high-frequency noise, making their direct use more critical for recognizing spurious events such as collisions. On the other hand, the currents follow approximately the shape of the commanded velocity (rather than the acceleration profile associated to the reference motion), thus confirming that the robot under low-level feedback behaves essentially as a firstorder system (with some disturbance and noise), justifying the use of purely kinematic control laws.

\section{A. Gravity identification}

In order to specify more stringent thresholds on motor currents so as to improve sensitivity of collision detection, we have performed an identification of the currents needed to sustain gravity in the different robot configurations. This gravity contribution is then eliminated from the measured currents before any filtering of the signals (see Sect. III-A).

Identification of the configuration-dependent gravity term $\boldsymbol{g}(\boldsymbol{q})$ is a subtask of the robot dynamic model identification problem [22], which can be addressed using by-now standard

\footnotetext{
${ }^{2}$ The proposed methods work also for the full $6 \mathrm{R}$ robot. For compactness, only the results on the first three joints are presented throughout the paper.

${ }^{3}$ For the sake of presentation, the position of joint 2 is shifted by $+90^{\circ}$ with respect to the D-H convention in Fig. 1.
}
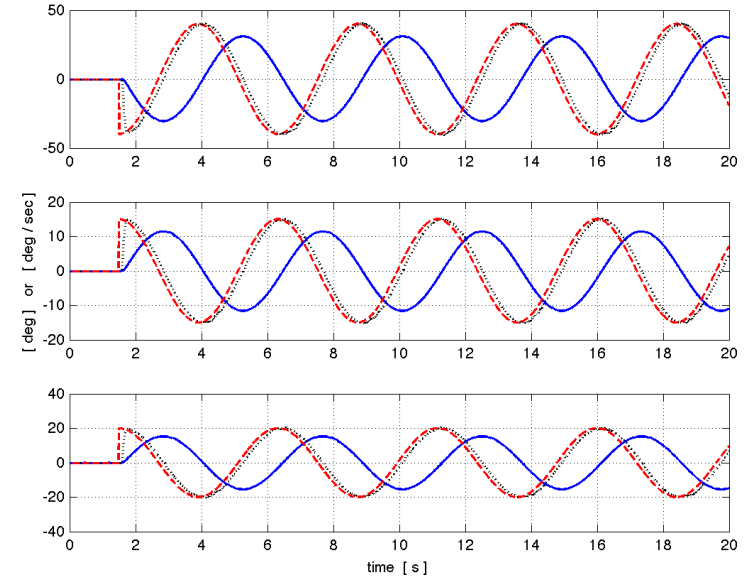

Fig. 3. Sinusoidal reference command velocity $\dot{\boldsymbol{q}}_{r}$ (dashed, red), measured position $\boldsymbol{q}$ (solid, blue), and velocity $\dot{\boldsymbol{q}}$ reconstructed numerically (dotted, black) for the first three joints of the KUKA KR5 robot
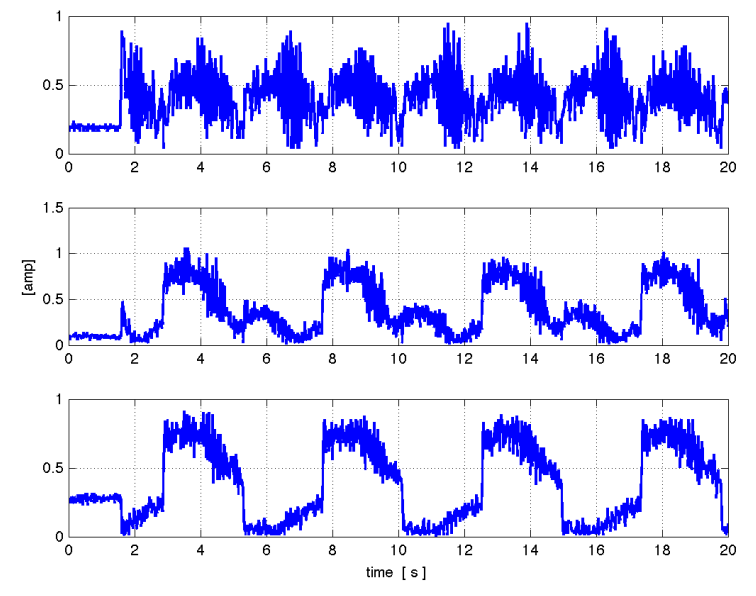

Fig. 4. Motor current signals for the first three robot joints on the motion of Fig. 3, as made available by the KUKA RSI interface

techniques based on joint torque (viz., current) and position measurements. However, in the present case we have to deal explicitly also with the lack of knowledge about the currentto-motor gains $\boldsymbol{K}_{i}$ and with the fact that only the absolute values of the motor currents $i$ are available.

The dynamic model of an electrically-driven robot manipulator with $N$ joints takes the usual form

$$
\boldsymbol{M}(\boldsymbol{q}) \ddot{\boldsymbol{q}}+\boldsymbol{c}(\boldsymbol{q}, \dot{\boldsymbol{q}})+\boldsymbol{f}(\boldsymbol{q}, \dot{\boldsymbol{q}})+\boldsymbol{g}(\boldsymbol{q})=\boldsymbol{\tau}
$$

with

$$
\boldsymbol{\tau}=\boldsymbol{K}_{\boldsymbol{i}} \boldsymbol{i}=\left(\begin{array}{lll}
k_{\boldsymbol{i}, 1} i_{1} & \ldots & k_{\boldsymbol{i}, N} i_{N}
\end{array}\right)^{T} .
$$

In static conditions, and neglecting the friction term $\boldsymbol{f}(\boldsymbol{q}, \dot{\boldsymbol{q}})$, one can proceed with the identification of the motor current $i_{g}$ associated to gravity by using its linear parametrized form,

$$
\boldsymbol{g}(\boldsymbol{q})=\boldsymbol{Y}_{\boldsymbol{g}}(\boldsymbol{q}) \boldsymbol{\theta}_{\boldsymbol{g}}=\left(\begin{array}{c}
\boldsymbol{y}_{1}^{T}(\boldsymbol{q}) \\
\vdots \\
\boldsymbol{y}_{N}^{T}(\boldsymbol{q})
\end{array}\right) \boldsymbol{\theta}_{\boldsymbol{g}}=\boldsymbol{K}_{\boldsymbol{i}} \boldsymbol{i}_{\boldsymbol{g}}
$$

where both the vector of dynamic coefficients $\boldsymbol{\theta}_{\boldsymbol{g}} \in \mathbb{R}^{M}$ and the square diagonal matrix $\boldsymbol{K}_{i}$ of size $N$ are unknown, and 
the gravity regressor matrix $\boldsymbol{Y}_{\boldsymbol{g}}$ has been expressed in terms of its rows $\boldsymbol{y}_{j}^{T}, j=1, \ldots, N$. Equation (3) can be rewritten component-wise as

$$
\boldsymbol{y}_{j}^{T}(\boldsymbol{q}) \frac{\boldsymbol{\theta}_{\boldsymbol{g}}}{k_{\boldsymbol{i}, j}}= \pm\left|i_{\boldsymbol{g}, j}\right|, \quad j=1, \ldots, N,
$$

where we made explicit the fact that only absolute values of the motor currents are available. In vector form, we obtain

$$
\boldsymbol{Y}(\boldsymbol{q}) \boldsymbol{\theta}=\boldsymbol{i}_{\boldsymbol{g}}^{ \pm}
$$

leading to $N$ linear equations in the $N \times M$ unknowns $\boldsymbol{\theta}$, with

$$
\begin{aligned}
& \boldsymbol{Y}(\boldsymbol{q})=\text { block } \operatorname{diag}\left\{\boldsymbol{y}_{j}^{T}(\boldsymbol{q})\right\} \\
& \boldsymbol{\theta}=\left(\begin{array}{c}
\boldsymbol{\theta}_{\boldsymbol{g}} / k_{\boldsymbol{i}, 1} \\
\vdots \\
\boldsymbol{\theta}_{\boldsymbol{g}} / k_{\boldsymbol{i}, N}
\end{array}\right), \quad \boldsymbol{i}_{\boldsymbol{g}}^{ \pm}=\left(\begin{array}{c} 
\pm\left|i_{\boldsymbol{g}, 1}\right| \\
\vdots \\
\pm\left|i_{\boldsymbol{g}, N}\right|
\end{array}\right) .
\end{aligned}
$$

When moving just the first three joints of our KUKA KR5 robot, gravity is present only at the second and third joints $(N=2)$. These terms can be parametrized with $M=3$ dynamic coefficients, depending on the mass and center of mass parameters of link 2 and of the composition of links 3 to 6 (the last three joints are kept in their zero configuration). We gathered static data from $P=20$ different robot configurations $\boldsymbol{q}_{\text {test }}$, in which the \pm signs of the joint torques (and thus of the holding currents) due to gravity is known from physical observation. The robot was moved to these configurations from opposite directions, so as to average the effects of static friction at steady state. The set of $P \times N$ eqs. (4) is solved then by pseudoinversion.

Once $\boldsymbol{\theta}$ has been estimated, we can remove at a generic configuration $\boldsymbol{q}$ the gravity components from the absolute values of the measured motor currents, using the absolute value of the left-hand side of eq. (4). Table I shows the results of the identification of gravity effects on motor currents in two validation experiments. The obtained accuracy is acceptable for the purpose of improving collision detection.

TABLE I

EVALUATION OF GRAVITY IDENTIFICATION ON MOTOR CURRENTS

\begin{tabular}{|c|c|c|c|c|}
\hline & \multicolumn{2}{|c|}{ current at joint 2 } & \multicolumn{2}{c|}{ current at joint 3 } \\
\hline test \# & measured & estimated & measured & estimated \\
\hline 1 & 0.77 & 0.76 & 0.1 & 0.05 \\
\hline 2 & -0.82 & -0.8 & -0.15 & -0.1 \\
\hline
\end{tabular}

\section{Collision Detection}

Force/torque exchanges at different locations of the robot body can arise because of an accidental collision with the human/environment or due to a desired physical collaboration between human and robot. Detection of these contacts with the robot is a fundamental feature for safe pHRI. A collision instantaneously generates torques at the robot joints, which in turn modify the planned robot motion. When the robot is controlled in a feedback mode (e.g., as in Fig. 2), the motor currents display then a sudden change, which is recognized as a collision when it exceeds some given threshold. This effect has been already used in the past for detecting collisions. We improve this basic signal-based method with the use of suitable filtering of the motor currents and by adopting trajectory-dependent thresholds. In doing so, it will also be possible to distinguish a collision from an intended contact, under the reasonable assumption that, for the latter case, the human is approaching and establishing physical contact with the robot in a softer way.

\section{A. Filtering of currents}

During a desired commanded motion of the KUKA KR5 robot, the absolute value of the currents at each motor is made available every $T=12 \mathrm{~ms}$ through the RSI interface. After removing from these signals the part needed for compensating gravity at the current configuration, as detailed in Sect. II-A, we propose the use of two filters working in parallel, namely a High-Pass Filter (HPF) and a Low-Pass Filter (LPF) of motors currents. The rationale is as follows:

- In most robot tasks, the desired motion is smooth and repetitive in nature and the frequency content of the associated commands (in feedback of feedforward mode) is limited and predictable in advance, especially in position-controlled robot like the KUKA KR5. On the other hand, noise as well as the effect of hard collisions typically appear in the high-frequency range of closedloop control signals.

- A LPF cleans the current signals from high-frequency noise, and possibly from the effect of hard collisions, while retaining the command frequencies needed for executing the motion task in a limited bandwidth. On the other hand, soft contacts between the robot and a human (intended for starting a collaboration) may be still recognized in the filtered signal.

- A HPF removes components that are slowly varying in time, down to constant offsets. The filtered current will still be very noisy, but is mostly sensitive to the effect of hard impacts (i.e., undesired/unexpected collisions).

Therefore, applying simultaneously a HPF and a LPF (or even multiple sets with different ranges of cutting frequencies) serves properly to our purposes. The filtering process is implemented on discrete-time data, and is the same for each motor current. Let $i_{k}=i\left(t_{k}\right)$, with $t_{k}=k T$, be the absolute value of the current at the sampled instant $t_{k}$ for a generic motor (as provided by the RSI) and let $i_{f, k}=i_{f}\left(t_{k}\right)$ be the output of the filter, with $f=\{\mathrm{LPF}, \mathrm{HPF}\}$.

The HPF was chosen as

$$
i_{\mathrm{HPF}, k}=h_{0} i_{k}+h_{2} i_{k-1}+h_{2} i_{k-2}+h_{3} i_{k-3},
$$

with $h_{0}=-0.239207, h_{1}=-0.6262528, h_{2}=0.6262528$, and $h_{3}=0.2392073$. Equation (5) represents a digital Chebyshev filter of order 3, with cutoff frequency equal to $10 \mathrm{~Hz}$. The order of the filter was limited to 3 to reduce as much as possible the delay on the output signal. The cutoff frequency was tuned based on the expected hardness/softness of impacts. For instance, a contact detected by a HPF with cutting frequency at $10 \mathrm{~Hz}$ but not detected when using the 
higher cutting frequency of $20 \mathrm{~Hz}$ is to be considered softer than one having effects also on the HPF at $20 \mathrm{~Hz}$.

For the LPF design, some initial trials with Chebyshev or Butterworth filters did not perform well, reducing too much the magnitude of the output signal. By analyzing the spectrum of typical input currents, we found that noise could be suppressed by filters with bandwidth near $20 \mathrm{~Hz}$, corresponding to a period of about $0.05 \mathrm{~s}$ (containing 4 samples). Therefore, we could remove effectively noise by choosing a simple average of three consecutive input samples, or

$$
i_{\mathrm{LPF}, k}=\frac{1}{3}\left(i_{k}+i_{k-1}+i_{k-2}\right) .
$$

\section{B. Thresholding}

The HPF and LPF currents are compared to thresholds in order to detect specific HRI events. To prevent false alarms or missed detections, time-varying thresholds $\tau_{\mathrm{HPF}}(t)$ and $\tau_{\mathrm{LPF}}(t)$ are specified on line depending on the commanded joint motion, namely on the reference velocity $\dot{\boldsymbol{q}}_{r}$ and on its acceleration $\ddot{\boldsymbol{q}}_{r}$ (obtained by backward numerical differentiation). This copes automatically with the different dynamic ranges of motion commands (being gravity contributions already eliminated from measured currents). Note that the use of commanded rather than actual (reconstructed) velocity $\dot{\boldsymbol{q}}$ avoids introducing unnecessary delays in the detection.

For a generic high-pass filtered motor current, we define

$$
\tau_{\mathrm{HPF}}=\tau_{H_{\text {min }}}+k_{H_{v}} \frac{\left|\dot{q}_{r}\right|}{v_{\max }}+k_{H_{a}} \frac{\left|\ddot{q}_{r}\right|}{a_{\max }}>0 .
$$

The first constant $\tau_{H_{\text {min }}}$ is chosen as the least value covering any HPF current in static conditions $\left(\dot{q}_{r}=\ddot{q}_{r}=0\right)$ at a joint configuration with zero gravity load. The robot joint is then run at its maximum velocity $v_{\max }>0$, and the gain $k_{H_{v}}>0$ is chosen so that the first two terms in the right-hand side of (7) provide an upper bound (with some margin) of the recorded motor currents. The same procedure is repeated in a similar way for the gain $k_{H_{a}}>0$, using the maximum acceleration $a_{\max }>0$. The value $\tau_{\mathrm{HPF}}$ will be the critical upper bound for the HPF current, while its opposite is taken as the critical lower bound.

For a generic low-pass filtered motor current, we have

$$
\tau_{\mathrm{LPF}}=\tau_{L_{\text {min }}}+k_{L_{v}} \frac{\left|\dot{q}_{r}\right|}{v_{\max }}+k_{L_{a}} \frac{\left|\ddot{q}_{r}\right|}{a_{\max }}>0,
$$

with similar definition and tuning of parameters as in eq. (7).

TABLE II

PARAMETERS OF THRESHOLDS ON FILTERED MOTOR CURRENTS

\begin{tabular}{|c|c|c|c|c|}
\hline \multicolumn{2}{|c|}{ joint } & 1 & 2 & 3 \\
\hline \multirow{3}{*}{ HPF } & $\tau_{H_{\min }}$ & 0.15 & 0.14 & 0.13 \\
& $k_{H_{v}}$ & 0.1 & 0.123 & 0.81 \\
& $k_{H_{a}}$ & 2.1 & 1.5 & 0.9 \\
\hline \multirow{3}{*}{ LPF } & $\tau_{L_{\min }}$ & 0.5 & 0.6 & 0.6 \\
& $k_{L_{v}}$ & 1.5 & 1.45 & 0.6 \\
& $k_{L_{a}}$ & 2.1 & 1.5 & 0.9 \\
\hline for both & $v_{\max }$ & 200 & 125 & 100 \\
HPF and LPF & $a_{\max }$ & 1200 & 1050 & 900 \\
\hline
\end{tabular}

In all presented experiments, we have used for eqs. (7) and (8) the parameter values specified in Tab. II. Figure 5
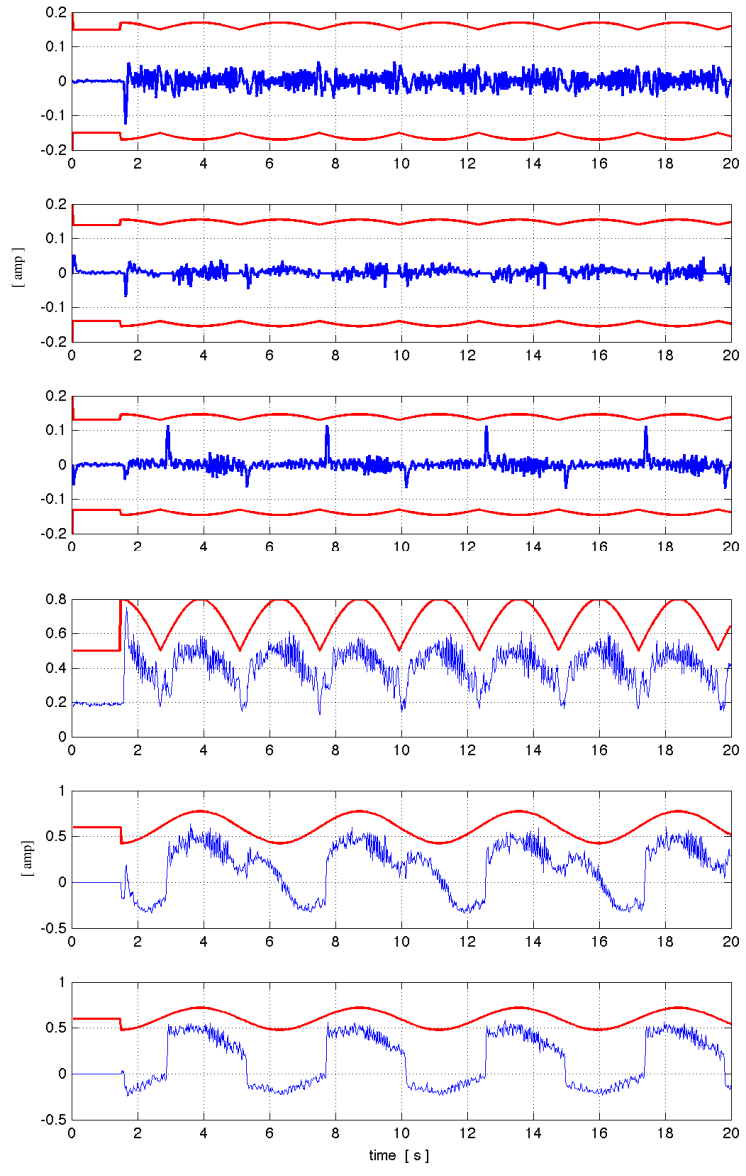

Fig. 5. High-pass [top] and low-pass [bottom] filtering of motor currents, with their time-varying thresholds. Case of no collisions during the robot motion of Fig. 3

shows the results obtained by HPF and LPF of the motor currents in Fig. 4, associated to the motion in Fig. 3, with the time-varying thresholds. In this case, no collisions occur and the thresholds are never reached. As opposed to the currents provided as output by the KUKA RSI interface (Fig. 4), the HPF and LPF currents take both positive and negative values (except for $i_{\mathrm{LPF}, 1}$, where gravity does not act and eq. (6) preserves positivity).

\section{Distinguishing collisions from intentional contacts}

We are now in place to formulate our simple rules for detection of accidental collisions and for detection of intentional contacts, distinguishing these two instances of pHRI.

Rule 1. A collision is detected if at least one HPF current exceeds its threshold.

Rule 2. An intentional contact is detected if no HPF current exceeds its threshold and at least one LPF current exceeds its threshold.

We present the results of a first experiment in which the two situations occur. The robot is continuously executing a given cyclic motion task defined in the joint space, when the human hits the robot body at different places (collisions). Each time a collision is detected (Rule 1), the robot stops and resumes then its motion after about $3 \mathrm{~s}$. Later on, the 
human seeks a soft contact with the robot, which is detected using Rule 2. In this experiment, no reaction is commanded and the robot continues its motion without changes.
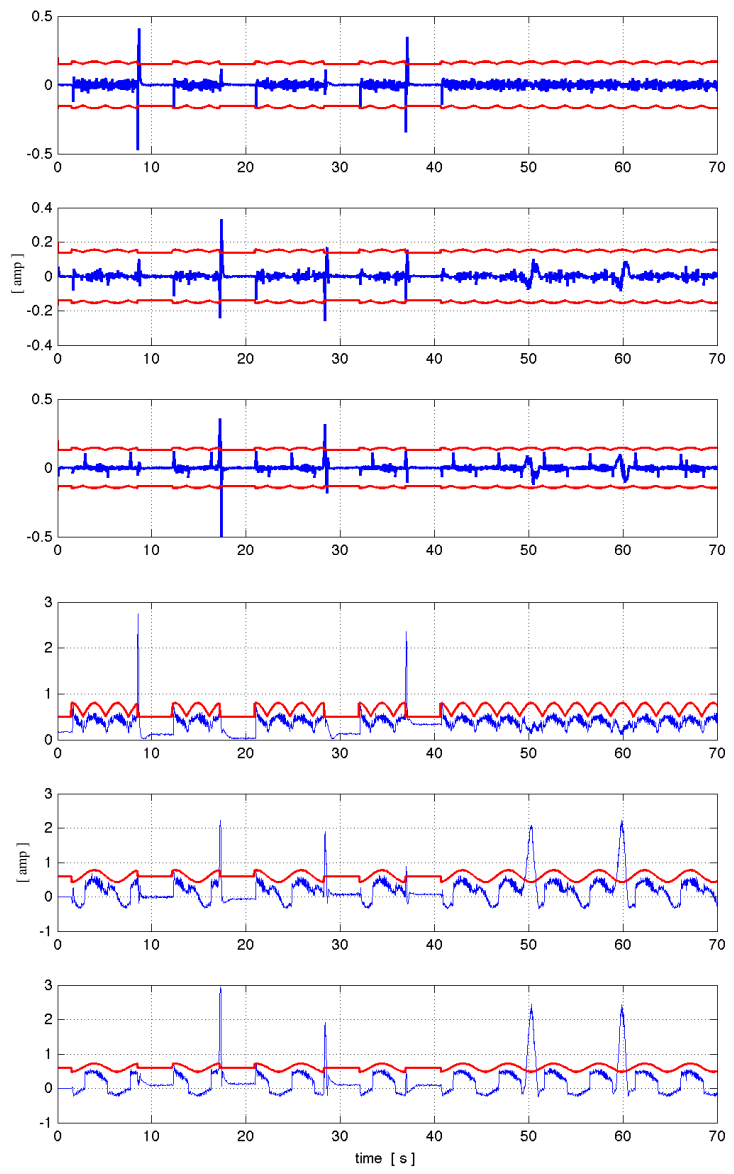

Fig. 6. High-pass [top] and low-pass [bottom] filtered motor currents on the robot motion in Fig. 7. Four collisions and then two intentional contacts are detected

Figure 6 shows the behavior of the HPF and LPF currents. Four collisions are detected, at about $t=8, t=17, t=28$, and $t=36 \mathrm{~s}$; two intentional contacts are detected at about $t=50$ and $t=60 \mathrm{~s}$. The joint motion $\boldsymbol{q}$, as measured by the robot encoders, and the associated kinematic control commands (the velocity reference $\dot{\boldsymbol{q}}_{r}$ ) are shown in Fig. 7 .

\section{Robot REACTION IN COLlaboRATION}

In our pHRI control framework, once a soft contact is (physically) requested by the human and detected by the combined use of LPF and HPF motor currents, the robot switches to a collaboration mode. In this mode, the robot can react to contact forces applied by the user in a variety of ways, e.g., by keeping its end-effector (or another part of its body) fixed in place or by moving in response to further contacts so as to approximately zeroing the forces exchanged with the human. Such behaviors are relatively easy to be realized on torque-controlled robots equipped with joint torque sensors and/or a $\mathrm{F} / \mathrm{T}$ sensor on the end-effector. For instance, when the human applies a force $f_{K}$ to a robot link, the resulting torque $\boldsymbol{\tau}_{K}=\boldsymbol{J}_{K}^{T}(\boldsymbol{q}) \boldsymbol{f}_{K}$ acting on the
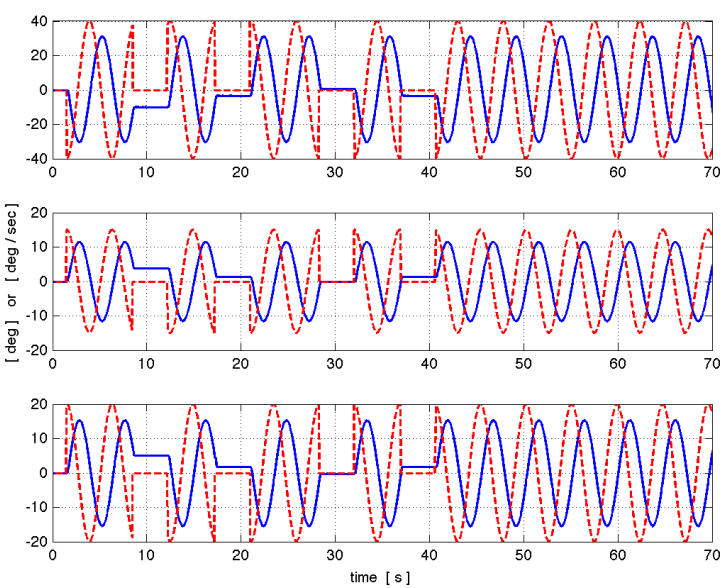

Fig. 7. Joint position measurements (solid, blue) and velocity reference commands (dashed, red) in a case of collisions and intentional contacts. In the first case the robot stops for $3 \mathrm{~s}$, while in the second no specific reaction is commanded

joints (being $\boldsymbol{J}_{K}$ the Jacobian associated to the unknown contact point) can be measured by the joint torque sensor, or estimated with a model-based residual as in [16], when the robot dynamic model is available. This torque $\boldsymbol{\tau}_{K}$ will indicate the direction in which the robot should move in the joint space, as an active response to the applied $\boldsymbol{f}_{K}$.

Unfortunately, no such concepts can be used directly on a standard industrial manipulator with closed control architecture, no extra sensors, and without a reliable dynamic model being available. In particular, having access only to the reference velocity $\dot{\boldsymbol{q}}_{r}$, we need to overcome (or trick) the relatively stiff embedded low-level controller in order to implement these robot reactions. In addition, in the case of our KUKA KR5 robot, the intrinsic uncertainty about the sign of the actual motor currents in the available measures makes it hard to understand —at least when using only these current signals - which is the direction of joint motion for properly reacting to an external force. Our idea to address this issue is to detect small initial variations of the joint position, as measured by the encoders, with respect to the desired value commanded by the low-level controller so as to recognize the joint-space direction for robot reaction (a similar method was used also in [20]). Based on this, we have realized a number of simple reactive strategies (see the full set of experiments in the video attachment, containing also other reactive behaviors).

\section{A. Human pushing/pulling the robot}

With the robot at rest and in collaboration mode, the human can apply continuous forces at any location of the robot and manually drive it to a desired configuration. The event is triggered by the LPF currents (after elimination of the components due to gravity). Looking at the (tiny) variation of joint positions (initially under the action of the KUKA controller with $\dot{\boldsymbol{q}}_{r}=\mathbf{0}$ ), a sign function $f_{j}=\{+,-\}$ is determined for each of the joints. These collectively specify the actual direction that will be taken by the robot in response to the force applied by the human. For this manual 

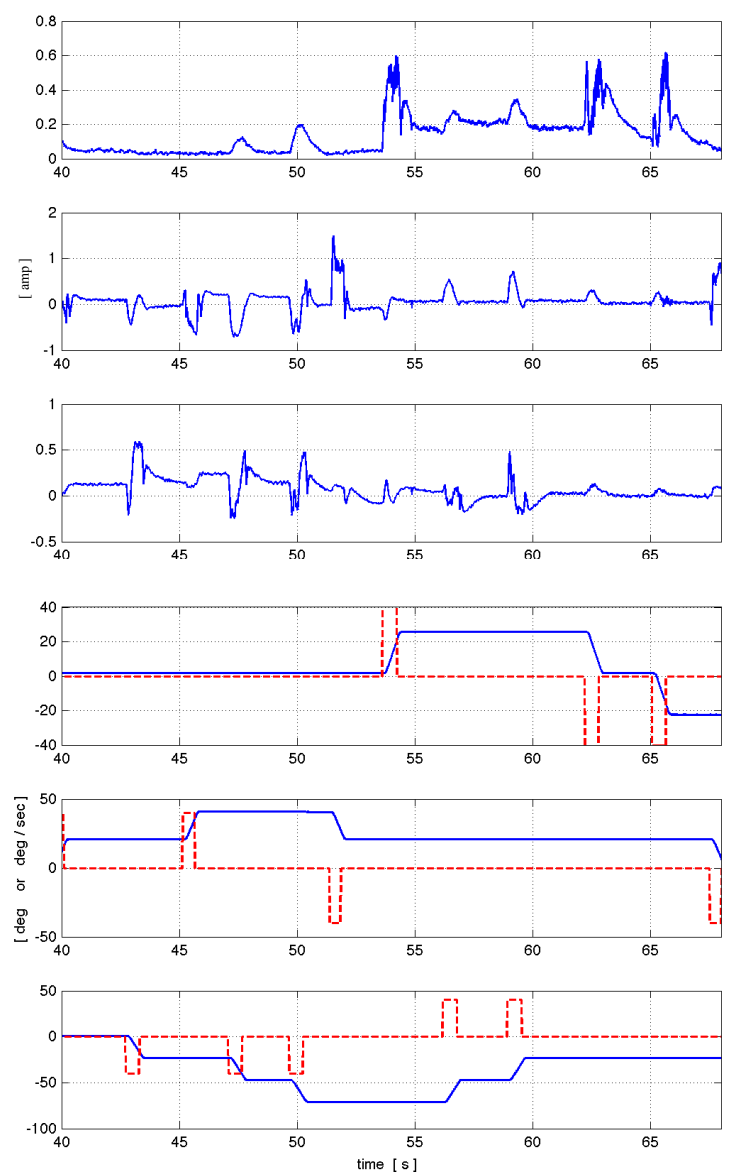

Fig. 8. LPF motor currents [top] and joint positions (solid, blue) and reference velocity commands (dashed, red) [bottom] for a collaboration mode where the human is pushing/pulling the robot with small forces (Experiment 3)

guidance mode, the control command $\dot{\boldsymbol{q}}_{r}$ is chosen as

$$
\dot{q}_{r, j}=f_{j} r_{j} i_{L P F, j}, \quad \text { for } j=1,2,3,
$$

where $r_{j}>0$ is an arbitrary gain to be tuned. Due to lack of space, the results are shown only in the accompanying video (Experiment 2).

In a second similar reaction strategy, the human can push/pull the robot away from the current configuration by applying even small but impulsive forces to the robot. Triggering of this event and finding the direction of robot reaction are the same as above, but the control command $\dot{\boldsymbol{q}}_{r}$ in this case is obtained by removing the presence of LPF currents from eq. (9). As a result, the joint velocity command will be constant, and its value is kept for some desired number of samples. Figure 8 shows the results obtained using $r_{j}=40$ for all joints. One can recognize the association between larger peaks in the LPF current profiles and constant commanded reference velocities. These plots correspond to the pushing/pulling Experiment 3 in the video.

\section{B. Compliant-like robot behavior}

At a given initial robot configuration $\boldsymbol{q}_{d}$, the human applies an instantaneous force to the robot. A compliant-like robot
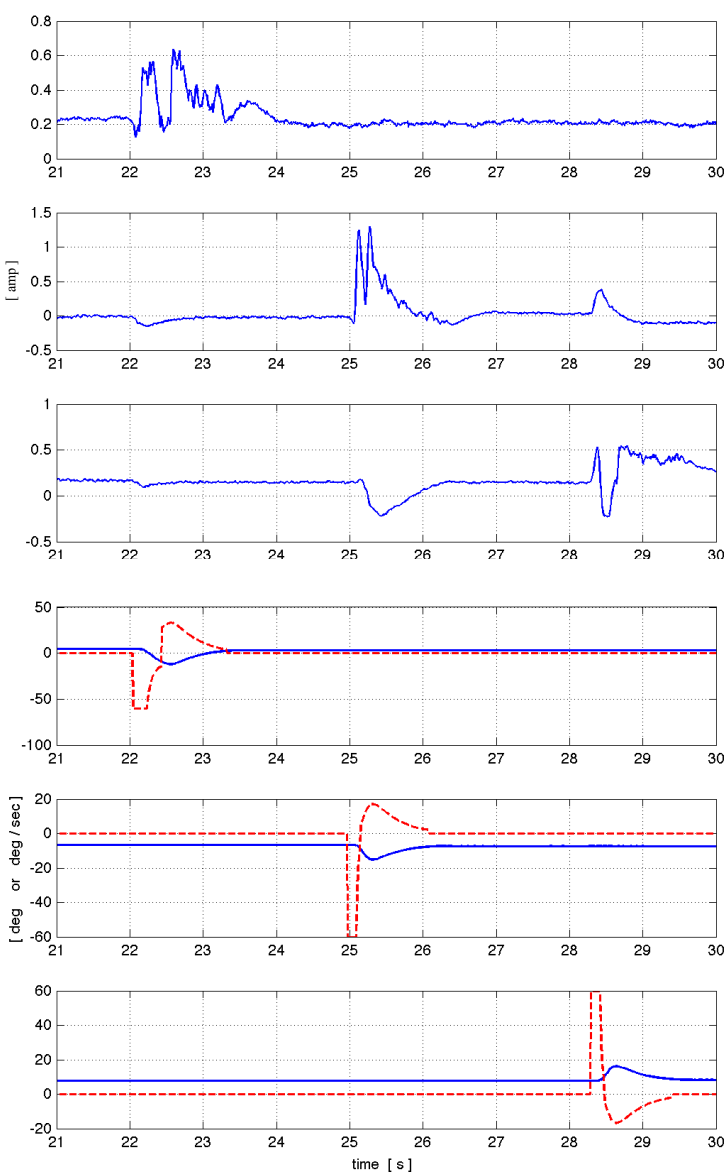

Fig. 9. LPF motor currents [top] and joint positions (solid, blue) and reference velocity commands (dashed, red) [bottom] for a collaboration mode where the robot shows a compliant-like behavior in response to instantaneous forces applied by the human (Experiment 4)

behavior is realized at the joint level by relating the control command $\dot{\boldsymbol{q}}_{r}$ to the joint error $\boldsymbol{e}=\boldsymbol{q}_{d}-\boldsymbol{q}$ and to the reaction direction in the form

$$
\dot{q}_{r, j}=f_{j} k_{j}+k_{p_{j}} e_{j}, \quad \text { for } j=1,2,3,
$$

where $k_{j}>0$ and $k_{p_{j}}>0$ are gains to be tuned for performance, and $f_{j}$ is defined as in Sect. IV-A. The two terms in (10) have opposite signs by construction, the first being constant while the latter is progressively increasing. At some instant, the two terms will balance each other and the robot will stop. In practice, when $\left|\dot{q}_{r, j}\right| \leq \epsilon_{j}$, being $\epsilon_{j}$ a small positive value, the first term is removed (setting $k_{j}=0$ from there on) and the second term will bring the robot back to the initial configuration $\boldsymbol{q}_{d}$. Figure 9 shows the results of a compliant collaboration mode obtained using $k_{j}=100$ and $\epsilon_{j}=0.01 \mathrm{deg}$ for all joints, $k_{p_{1}}=6, k_{p_{2}}=k_{p_{3}}=11.8$, while setting in the second reaction phase the compliant gains $k_{p_{i}}=2$ for all joints. A rapid and smooth recover of the initial configuration is obtained (see Experiment 4 in the video). 


\section{COnClusions}

We have presented a signal-based approach to wholebody collision detection, robot reaction, and human-robot collaboration that works for industrial manipulators with a closed control architecture and without the use of extra sensors (e.g., joint torque or 6D F/T sensors). The signals used are those available to a generic end-user through the data interface provided by the manufacturer: joint velocity reference (as control input to the system), joint position (as measured output), and a signal related to the internal motor currents. In this framework, we introduced the idea of highpass and low-pass filtering of currents. By applying simple rules on these two filtered measures, one can distinguish between accidental hard collisions and intentional soft contacts between the human and the robot. In response to a detected intentional contact, some examples of robot reactive behavior in collaboration mode were provided, including manual robot guidance, pushing/pulling the robot by instantaneous forces, and a compliant-like robot behavior in response to small forces. In all cases, contact forces can be applied by the human at any point of the robot structure.

The methods have been implemented on a KUKA KR5 robot using the RSI interface. With the communication and control rates allowed by the RSI, achieving high robot performance is indeed difficult. Although no ground truth measure of the actual instant of human-robot collision and of the minimum detectable collision force (needing an external force measurement) are available at this time, we estimate that collisions producing more than $0.2 \mathrm{~A}$ in at least one of the HPF motor currents can be safely detected, with collision detection times of the order of 36-48 ms (3-4 sampling intervals). For comparison, in our previous experience with the DLR LWR-III [16], [17] (or, equivalently, with the KUKA LWR4+ and its $1 \mathrm{~ms}$ Fast Research Interface) we reached detection times of the order of 2-3 ms.

While we achieved very good subjective results both in collision detection and in distinguishing intentional from accidental collisions, our future plan is to conduct a statistical analysis of the sensitivity and robustness of the classification, using a set of volunteers and a suitable experimental protocol. Moreover, some of the introduced ideas, like using LPF and HPF, are general and can be applied also to process residual signals in torque-controlled robots with an accurate dynamic model available, so as to distinguish collisions from intentional contacts at a faster rate. Another on-going work along the development of safe human-robot co-existence and collaboration includes the use of exteroceptive sensors and their integration with basic collision detection and reaction strategies. For instance, a Kinect can be used for collision avoidance but also to track the body parts of a human physically interacting with the KUKA KR5 robot (see Experiment 5 in the video attachment). This allows locating the area of human contact on the robot (with its associated Jacobian), and the implementation of more sophisticated reaction/force control schemes based on the actual contact point.

\section{REFERENCES}

[1] J. Heinzmann and A. Zelinsky, "Quantitative safety guarantees for physical human-robot interaction," Int. J. of Robotics Research, vol. 22 , no. 7/8, pp. 479-504, 2003.

[2] A. De Santis, B. Siciliano, A. De Luca, and A. Bicchi, "An atlas of physical human-robot interaction," Mechanism and Machine Theory, vol. 43, no. 3, pp. 253-270, 2008.

[3] S. Haddadin, A. Albu-Schäffer, and G. Hirzinger, "Requirements for safe robots: Measurements, analysis and new insights," Int. J. of Robotics Research, vol. 28, no. 11/12, pp. 1507-1527, 2009.

[4] A. Bicchi, M. A. Peshkin, and J. E. Colgate, "Safety for physical human-robot interaction," in Springer Handbook of Robotics, B. Siciliano and O. Khatib, Eds. Springer, 2008, pp. 1335-1348.

[5] G. Hirzinger, A. Albu-Schäffer, M. Hähnle, I. Schaefer, and N. Sporer, "On a new generation of torque controlled light-weight robots," in Proc. IEEE Int. Conf. on Robotics and Automation, 2001, pp. 33563363.

[6] K. Salisbury, W. Townsend, B. Eberman, and D. DiPietro, "Preliminary design of a whole-arm manipulation system (WAMS)," in Proc. IEEE Int. Conf. on Robotics and Automation, 1988, pp. 254-260.

[7] A. De Luca and W. Book, "Robots with flexible elements," in Springer Handbook of Robotics, B. Siciliano and O. Khatib, Eds. Springer, 2008, pp. 287-319.

[8] A. Kugi, C. Ott, A. Albu-Schäffer, and G. Hirzinger, "On the passivitybased impedance control of flexible joint robots," IEEE Trans. on Robotics, vol. 24, no. 2, pp. 416-429, 2008.

[9] R. Schiavi, G. Grioli, S. Sen, and A. Bicchi, "VSA-II: A novel prototype of variable stiffness actuator for safe and performing robots interacting with humans," in Proc. IEEE Int. Conf. on Robotics and Automation, 2008, pp. 2171-2176.

[10] S. Kuhn, T. Gecks, and D. Henrich, "Velocity control for safe robot guidance based on fused vision and force/torque data," in Proc. IEEE Int. Conf. on Multisensor Fusion and Integration for Intelligent Systems, 2006, pp. 485-492.

[11] S. Kuhn and D. Henrich, "Fast vision-based minimum distance determination between known and unknown objects," in Proc. IEEE/RSJ Int. Conf. on Intelligent Robots and Systems, 2007, pp. 2186-2191.

[12] F. Flacco, T. Kröger, A. De Luca, and O. Khatib, "A depth space approach to human-robot collision avoidance," in Proc. IEEE Int. Conf. on Robotics and Automation, 2012, pp. 338-345.

[13] K. Suita, Y. Yamada, N. Tsuchida, K. Imai, H. Ikeda, and N. Sugimoto, 'A failure-to-safety 'kyozon' system with simple contact detection and stop capabilities for safe human-autonomous robot coexistence," in Proc. IEEE Int. Conf. on Robotics and Automation, 1995, pp. 30893096.

[14] Collision Detection RobotWare option, ABB Robotics Products, SE72168 Västerås, Sweden, 2008, PR10044en, Version 2.1.

[15] S. Morinaga and K. Kosuge, "Collision detection system for manipulator based on adaptive impedance control law," in Proc. IEEE Int. Conf. on Robotics and Automation, 2003, pp. 1080-1085.

[16] A. De Luca, A. Albu-Schäffer, S. Haddadin, and G. Hirzinger, "Collision detection and safe reaction with the DLR-III lightweight robot arm," in Proc. IEEE/RSJ Int. Conf. on Intelligent Robots and Systems, 2006, pp. 1623-1630.

[17] S. Haddadin, A. Albu-Schäffer, A. De Luca, and G. Hirzinger, "Collision detection and reaction: A contribution to safe physical humanrobot interaction," in Proc. IEEE/RSJ Int. Conf. on Intelligent Robots and Systems, 2008, pp. 3356-3363.

[18] M. Erden and T. Tomiyama, "Human-intent detection and physically interactive control of a robot without force sensors," IEEE Trans. on Robotics, vol. 26, no. 2, pp. 370-382, 2010.

[19] A. De Luca and F. Flacco, "Integrated control for pHRI: Collision avoidance, detection, reaction and collaboration," in Proc. 4th IEEE RAS \& EMBS Conf. on Biomedical Robotics and Biomechatronics, 2012, pp. 288-295.

[20] A. Stolt, M. Linderoth, A. Robertsson, and R. Johansson, "Force controlled robotic assembly without a force sensor," in Proc. IEEE Int. Conf. on Robotics and Automation, 2012, pp. 1538-1543.

[21] KUKA.RobotSensorInterface (RSI), KUKA System Technology (KST), D-86165 Augsburg, Germany, 2007, revision 2.

[22] J. Swevers, W. Verdonck, and J. De Schutter, "Dynamic model identification for industrial robots," IEEE Control Systems Mag., vol. 27, no. 5, pp. 58-71, 2007. 